\title{
HCI Standards for Handicapped
}

\author{
Zbigniew Wisniewski and Aleksandra Polak-Sopinska \\ Department of Production Management, Technical University of Lodz, \\ ul. Wolczanska 215, 90-924 Lodz, Poland \\ \{zwisniewski, olapolak\}@p.lodz.pl
}

\begin{abstract}
The study presents conclusions from the project realization so far. The main aim of the project is to work out the rules of evaluation and validation of application interfaces on the basis of study of the process of man-computer communication. The target group for the benefit of which the research will be carried out are disabled persons and people over 55 years of age. The disabled and the elderly, are in the social stratum systematically ignored by software producers and internet service providers as a target group of computer users.
\end{abstract}

Keywords: HCI, elderly, disabled people, digital divide.

\section{Introduction}

The term digital divide refers to a rapidly extending gap between Internet users and non-users. According to research about $40 \%$ of Europeans does not use the Internet in their daily life. There are a variety of reasons, including lack of funds, limited availability of the network, insufficient computer literacy. Less than $12 \%$ of people over 65 use the Internet, which is associated with the fact that only $3 \%$ of the websites meets the accessibility standards. The issue of no accessibility in the first place refers to people with various dysfunctions of the organ of sight. In most cases website designers are not aware of the difficulties the blind and sand-blind people encounter when trying to use the Internet.

The ageing process has become so common in many countries that it is reflected in virtual world as well, since older people constitute an increasing number of Internet users. The popularity of life-long learning is growing, too. Many older people want to learn new things and acquire new skills, develop themselves and fully participate in everyday life at the same time fighting with weaknesses.

The above tendency results in implementation of Internet usage facilities aimed at older and disabled people. In European Union countries approximately 10-15\% of the whole population is disabled people with various types of dysfunctions. The report published by the United Nations Organizations shows that in 2050 almost two billion people will be over 60, whereas nowadays the number of people over 60 does not exceed 800 million. The Internet is a great opportunity for the disabled and the elderly, who cannot be fully active due to some limitations. Internet access facilitates independence, contact with other people, the ability to do some everyday activities unassisted. 
All efforts notwithstanding, older and disabled people still encounter a number of barriers. These are mainly due to some technical restrictions of designed websites and applications. In this case the significance of accessibility is undeniable. Applications and websites designed in accordance with accessibility principles enable their easy and friendly use by the disabled.

\section{Standards}

W3C (World Wide Web Consortium) - international consortium with more than 400 members representing some of the biggest and most renowned companies, institutions, organizations and universities from all over the world. The goal of W3C is to create Web standards. W3C recommendations are the guidance for the whole civilized world.

Since 1997 W3C has been running an activity called Web Accessibility Initiative (WAI), embracing a group of people and organizations engaged in development and promotion of Internet (mainly website) accessibility standards aimed at disabled people. Among the participants of this initiative there are such organizations as IBM, Microsoft, Bell Atlantic, Lotus Development Corporation, the government of Canada, National Institute on Disability and Rehabilitation Research at the American Department of Education and European Union representatives. WAI has created the following sets of guidelines.

Authoring Tool Accessibility Guidelines - a set of guidelines for authors designing Web content. In order to enable disabled people to use the Internet to its full potential it is essential to help them publish their own materials. Thus software for creating websites should provide facilities for the disabled. The updated version of the guidelines is currently being prepared.

- User Agent Accessibility Guidelines - guidelines on designing web browsers that satisfy the requirements of people with disabilities.

- XML Accessibility Guidelines. XML is a standard devised by W3C, which focuses on storage of all types of data. Due to its expansible nature it is necessary that authors of new languages based on XML do not impede accessibility.

- Web Content Accessibility Guidelines (WCAG) - guidelines on designing websites that satisfy the requirements of people with disabilities. Since 1999 WCAG 1.0 has been the official $\mathrm{W} 3 \mathrm{C}$ recommendation. Currently the second updated version of this standard is being prepared. WCAG guidelines have become the basis for accessibility regulatory requirements for many European Union members and other countries. Given the above, it is worth taking a closer look at the guidelines. The main goals of WCAG include: providing access to Web content to users of various devices (text and graphic web browsers, voice browsers, portable devices), logic and readable content organization as well as clear and convenient navigation.

\section{Research and Findings}

The scope of research included identification of groups of users who have difficulties with the Internet due to the fact that the interfaces are not adjusted to their specific needs. These people are able to use peripheral devices (keyboard, mouse, and monitor): 
- people with motor disabilities:

- upper limb dysfunctions restricting the ability to use mouse and keyboard,

- problems with long-term remaining in sitting position,

- people with ADHD,

- people with moderate mental disability,

- people with moderate impairment of the organ of sight,

- people with defective perception of colours,

- people with epilepsy,

- people with dyslexia,

- older people (55+),

The research on Web accessibility, functionality and usability is carried out by means of special applications. The first application is aimed at testing and identifying the needs of users with specific perceptual-motor dysfunctions. The application is based on the following pattern:

- generating a website with features required by the program in order to have composition tested by the users,

- gathering results in the data base,

- statistic processing,

- reporting.

The research is carried out among organizations grouping older people willing to use the Internet: universities of the Third Age, senior clubs, etc.

The second application is used for a wide-sample research and is made up of the following stages:

1. Gathering user information,

2. Gathering web browser information,

3. Gathering website content information:

- HTML (controls and their parameters, other elements and their parameters colour, typography),

- Text statistics,

- Picture analysis- colour, text contrast, text size, detection and analysis of composition of typical website elements),

4. Sending report to the data base.

This application has the form of a FireFox browser plug-in. It concentrates on gathering data on user activity and their opinion of visited websites. The data refer to how users deal with a website and what operations they perform. During these observations the ability to perform particular operations is being assessed (the number of repetitions, the number of failed operations, and the time spent on particular operations, etc.) Moreover, the users are occasionally asked to express their opinion and provide some information, including their disability, if applicable.

Both tools enable better identification of the special needs disabled people have when using the Internet. The first application helps recognize perceptual-motor 
abilities of the focus group. The second application is used to verify the results of the first one as well as to extent the scope of exploration. In the first case the application generates assigned patterns in the form of interfaces and the participants are aware of the test, whereas in the second case the research is carried out on real websites. Due to the fact that it is performed via a browser plug-in the participants are not 'tempted' to show off and behave more naturally and at ease than in the first case.

This last research provides some interesting findings on websites most frequently visited by people with dysfunctions. The users are able to select an area in the Net where they want to be active and simply perform typical activities. The findings provide some noteworthy information on Internet activity of various users and their perceptual-motor abilities. However, the final conclusions can be presented only after the research completion, bound next year.

For the time being the available conclusions seem to be in accordance with Web content creation guidelines published by the mentioned organizations. The guidelines are as follows:

1. sound and visual elements should have their alternative equivalents,

2. colour should not be the only means of distinguishing information,

3. technologies defining structure and presentation should be used,

4. language used in a website or its fragments should be constrained, which is absolutely necessary for proper functioning of screen readers,

5. tables describing website structure should be avoided, tables should only be used for comparisons or summaries, moreover they should be properly named so that screen readers are able to present their content to the users,

6. website content should be available to users not possessing the latest technology, e.g. script languages, plug-ins,

7. it should be possible to switch off or stop the animation (which is crucial for users suffering from epilepsy), animated elements should be avoided where possible,

8. objects located in the website structure (e.g. applets, scripts, etc.) should have interface available for a variety of manipulators (e.g. mouse, keyboard, etc.),

9. new technology implementation should be accompanied by temporary solutions to enable access to all users,

10. elements facilitating structure orientation (e.g. labels, summaries, etc.) should be used,

11. navigation should be consistent and clear, which assures good orientation in the website structure,

12. documents should be readable, clear and written in a simple language.

The most common errors of website designers include the tendency to use Flash and technology and DHTML elements much more than it is necessary. Obviously these technologies help create an attractive website; however, their excessive use leads to lowered accessibility for disabled people.

The most serious error of Web content authors is non conformity of the source code with the guidelines published by such organizations as World Wide Web Consortium or The Unicode Consortium.

The core principle of useful and accessible website designing is to follow the standards of competent organizations (e.g. W3C). Conforming to and following the standards maximizes accessibility of all websites for disabled people. This aspect of Web 
content design is particularly significant in case of e-learning platforms, websites of national organizations or banks.

\section{References}

1. Laurel, B.: The art of human-computer interface design. Addison-Wesley, Reading (1990)

2. Landauer, T.K.: The trouble with computers: Usefulness, usability, and productivity. MIT Press, Cambridge (1995)

3. Mullet, K., Sano, D.: Designing visual interfaces: Communication oriented techniques. Sunsoft Press/Prentice Hall, Englewood Cliffs (1995)

4. Raskin, J.: The humane interface: New directions for designing interactive systems. Addison-Wesley, Reading (2000)

5. Wiśniewski, Z.: Ergotronics - object, composition, message, transfer. In: Pacholski, L.M., Trzcieliński, S. (eds.) Ergonomics in contemporary enterprise. IEA Press Madison, Poznań (2007) 